\title{
Spectroscopy studies using two-nucleon knockout
}

\author{
J. A. Tostevin \\ Department of Physics, School of Electronics and Physical Sciences, University of Surrey, \\ Guildford GU2 7XH, United Kingdom
}

\begin{abstract}
Two-proton removal reaction cross sections, from ${ }^{208} \mathrm{~Pb}$ at $1 \mathrm{GeV} /$ nucleon, are estimated as an example of the direct population of (high-spin) seniority-2 isomeric states, here in ${ }^{206} \mathrm{Hg}$. Nucleon removal by both the stripping and diffractive mechanisms is considered. The cross sections in this specific (test) case are significant and can provide direct two-nucleon removal predictions of isomeric ratios.
\end{abstract}

Keywords: Direct reactions, two-proton knockout, seniority isomers, neutron-rich spectroscopy PACS: $21.10 . J x, 24.10 .-\mathrm{i}, 25.70 .-\mathrm{z}, 27.80 .+\mathrm{w}$

\section{INTRODUCTION}

One-nucleon, and selected two-nucleon knockout reactions from intermediate energy beams proceed as sudden, direct reactions $[1,2,3]$. When these are combined with coincident gamma-ray, or other final-state-selective detection of the reaction residues, the partial cross section measurements provide a demanding test of modern nuclear structure model predictions of one- and two-nucleon configurations in nuclei. To date, direct two-nucleon knockout reactions have been considered using uncorrelated, partially correlated (cluster) and fully-correlated (shell model) structure model descriptions, together with eikonal reaction theory $[2,3]$. Recent applications, to light and medium-mass nuclei, include two-proton removal from neutron-rich ${ }^{28} \mathrm{Mg}[2,3],{ }^{44} \mathrm{~S}$ [4] and ${ }^{54} \mathrm{Ti}$ [5] and two-neutron removal from the neutron-deficient ${ }^{34} \mathrm{Ar},{ }^{30} \mathrm{~S}$ and ${ }^{26} \mathrm{Si}$ [6]. The eikonal reaction theory is able to include both the elastic (diffraction) and inelastic (stripping) nucleon-removal mechanisms.

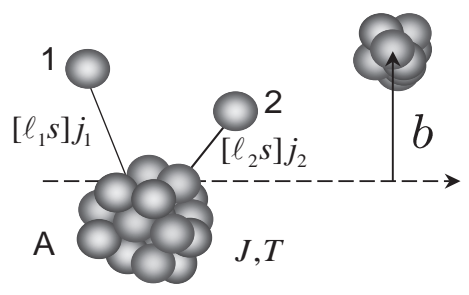

FIGURE 1. Schematic of the uncorrelated nucleons description of the two-nucleon knockout reaction.

Here, as a test case for heavier nuclei, we investigate the direct, two-proton removal cross sections from ${ }^{208} \mathrm{~Pb}\left(0^{+}\right)$. Final state $J^{\pi}$ selectivity is assumed and we consider the population of specific isomeric states in the ${ }^{206} \mathrm{Hg}$ residue. We assume, see Figure 1, 
the removal of the pair of nucleons from Hartree-Fock single-particle states $\phi_{j_{1}}$ and $\phi_{j_{2}}$. Thus, except for the spatial correlations due to the nucleons being bound to a common core, and those arising from antisymmetry and angular momentum coupling, the two protons are assumed to be uncorrelated. As was discussed in [2, 3], the stripping (inelastic breakup) cross section is then, with $\hat{J}^{2}=(2 J+1)$,

$$
\sigma_{j_{1} j_{2}}(J)=\frac{1}{\hat{J}^{2}} \sum_{M} \int d \vec{b}\left|\mathscr{S}_{c}\right|^{2}\left\langle\left[\overline{\phi_{j_{1}} \otimes \phi_{j_{2}}}\right]_{J M}\left|\left(1-\left|\mathscr{S}_{1}\right|^{2}\right)\left(1-\left|\mathscr{S}_{2}\right|^{2}\right)\right|\left[\overline{\phi_{j_{1}} \otimes \phi_{j_{2}}}\right]_{J M}\right\rangle .
$$

The integral is carried out over all projectile center-of-mass $(\mathrm{cm})$ impact parameters $\vec{b}$, see Figure 1 . The $\mathscr{S}_{i}(i=1,2, c)$ are the eikonal S-matrices [1] describing the interactions of the two nucleons $(1,2)$ and the $A$-body residue, or core $c$, with the target. Each $\mathscr{S}_{i}$ is a function of its own impact parameter $b_{i}$ and is assumed to be spin-independent. This expression reflects the stripping mechanism where the residue interacts at most elastically with the target, survives the collision, and escapes to infinity; reflected by $\left|\mathscr{S}_{c}\right|^{2}$. The two removed nucleons interact inelastically with the target and are absorbed from the elastic channel; seen by the product of their absorption probabilities $\prod_{i}\left(1-\left|\mathscr{S}_{i}\right|^{2}\right)$. Here we have made the (sudden) adiabatic and the eikonal (forward scattering) approximations, both expected to be excellent at the energies of interest. We have also made the spectator-core approximation, that dynamical excitation of the core during the collision can be neglected, as are the effects of recoil of the heavy mass $A$ residue, by setting $b_{c}=b$.

Eq. (1) has been applied to ${ }^{28} \mathrm{Mg}$ and other systems [2, 3]. Additional (diffractive) cross section enters the terms in the eikonal theory with the form

$$
\bar{\sigma}_{j_{1} j_{2}}(J)=\frac{1}{\hat{J}^{2}} \sum_{M} \int d \vec{b}\left|\mathscr{S}_{c}(b)\right|^{2}\left\langle\left.\left[\overline{\phi_{j_{1}} \otimes \phi_{j_{2}}}\right]_{J M}|| \mathscr{S}_{1}\right|^{2}\left(1-\left|\mathscr{S}_{2}\right|^{2}\right) \mid\left[\overline{\phi_{j_{1}} \otimes \phi_{j_{2}}}\right]_{J M}\right\rangle,
$$

where only one nucleon (here 2) is absorbed. These describe all processes where both nucleon 1 and the core emerge from the collision. These diffraction contributions are included here and are discussed in detail elsewhere [7]. The cross section for diffractive removal of both nucleons is negligible at the energy of interest. Reference to figure 2 suggests that indirect population of the ${ }^{206} \mathrm{Hg}$ final states of interest, by one-proton removal to states in the ${ }^{207} \mathrm{Tl}$ continuum and proton evaporation, is also expected to be negligible.

\section{DIRECT TWO-PROTON KNOCKOUT FROM ${ }^{208}$ PB}

We calculate two-proton knockout cross sections from a $1.0 \mathrm{GeV}$ per nucleon ${ }^{208} \mathrm{~Pb}$ beam incident on a ${ }^{9} \mathrm{Be}$ target. The required core- and proton-target S-matrices were calculated from the core and target point nucleon densities using the optical limit of Glauber's multiple scattering theory [8,9]. A zero-range nucleon-nucleon (NN) effective interaction was assumed with strength determined, in the usual way [10], by the free $\mathrm{pp}$ and $\mathrm{np}$ cross sections. The real-to-imaginary ratios of the forward scattering NN amplitudes were taken to be zero. The neutron and proton densities in ${ }^{206} \mathrm{Hg}$ were given by Hartree-Fock calculations [11], using the Skyrme SkP interaction [12], with rms radii 


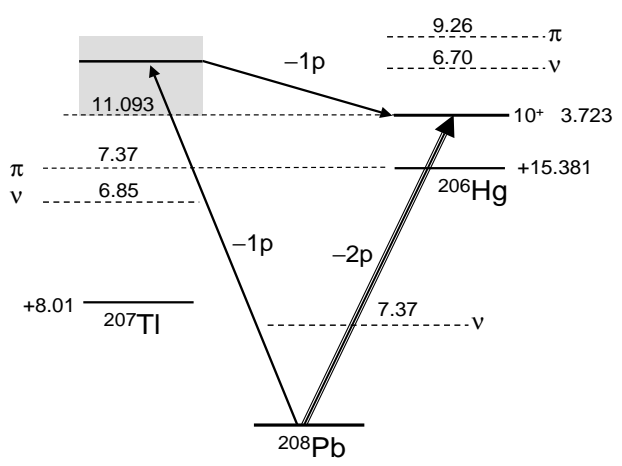

FIGURE 2. Schematic of the direct two-proton knockout reaction to the ${ }^{206} \mathrm{Hg}\left(10^{+}\right)$state, and the associated neutron and proton thresholds. Non-direct population of ${ }^{206} \mathrm{Hg}$ bound states, by one-proton removal to excited ${ }^{207} \mathrm{Tl}$ followed by proton evaporation, involves intermediate states above the (lower) neutron evaporation threshold and is expected to be small.

of $5.606 \mathrm{fm}$ and $5.445 \mathrm{fm}$, respectively. The density of the ${ }^{9} \mathrm{Be}$ target was assumed to be of Gaussian form with rms matter radius of $2.36 \mathrm{fm}$. However, analyses of onenucleon knockout reveal that calculations show little sensitivity to the details of these radial forms, e.g. [13].

The ${ }^{206} \mathrm{Hg}$ final states of interest, Figure 3, are the $\left(10^{+}\right)(3.723 \mathrm{MeV}),\left(8^{+}\right)$ $(3.623 \mathrm{MeV}),\left(7^{-}\right)(2.466 \mathrm{MeV})$ and $5^{-}(2.102 \mathrm{MeV})$ [seniority-2] two-proton hole states. These have dominant components with $\pi\left[0 h_{11 / 2}^{-2}\right], \pi\left[0 h_{11 / 2}^{-2}\right], \pi\left[0 h_{11 / 2}^{-1} 1 d_{3 / 2}^{-1}\right]$ and $\pi\left[0 h_{11 / 2}^{-1} 2 s_{1 / 2}^{-1}\right]$, respectively. The ${ }^{208} \mathrm{~Pb}$ ground state to ${ }^{206} \mathrm{Hg}$ ground state two proton separation energy is $15.381 \mathrm{MeV}$, Figure 2. Hence the separation energies to these $J^{\pi}\left(E^{*}\right)$ excited states are $\left[15.381+E^{*}\right] \mathrm{MeV}$. These yield $S_{2 p}\left(0 h_{11 / 2}^{2}\right) \approx 19.0$ $\mathrm{MeV}, S_{2 p}\left(0 h_{11 / 2} 1 d_{3 / 2}\right)=17.85 \mathrm{MeV}$ and $S_{2 p}\left(0 h_{11 / 2} 2 s_{1 / 2}\right)=17.50 \mathrm{MeV}$. We take, $S_{p}\left(0 h_{11 / 2}\right)=9.5 \mathrm{MeV}, S_{p}\left(1 d_{3 / 2}\right)=8.35 \mathrm{MeV}, S_{p}\left(2 s_{1 / 2}\right)=8.0 \mathrm{MeV}$, in agreement with the one proton separation energy and the ${ }^{207} \mathrm{Tl}$ spectrum.

The bound state proton-core single-particle wave functions were calculated in WoodsSaxon potential wells with a conventional diffuseness parameter, $a=0.70 \mathrm{fm}$. The potential radius parameters, $r_{0}$, were adjusted (for each single particle orbital) to reproduce the rms radius of the Hartree-Fock calculation [11]. These give $r_{0}=1.336 \mathrm{fm}, 1.282 \mathrm{fm}$ and $1.323 \mathrm{fm}$ for the $1 d_{3 / 2}, 0 h_{11 / 2}$ and $2 s_{1 / 2}$ states. The strength of the binding potentials were adjusted to reproduce the physical separation energies, as above. A 6.0 MeV spin-orbit potential, with the same geometry parameters as for the central potential, was included. Thus, both the Coulomb and centrifugal barriers experienced by the removed protons are included fully. Our results for the knockout of uncorrelated proton pairs are collected in Table 1 . 


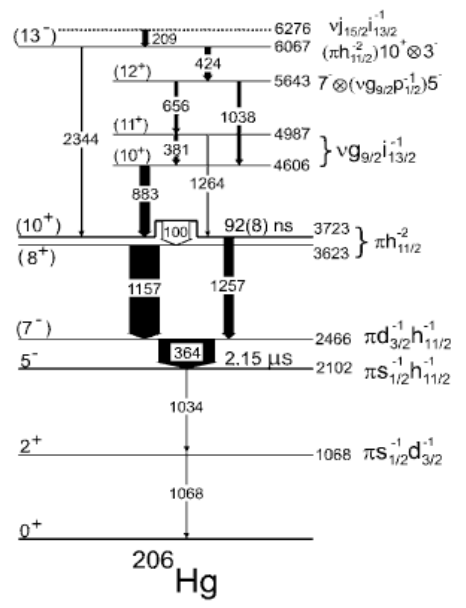

FIGURE 3. States in ${ }^{206} \mathrm{Hg}[14]$, showing the $\left(10^{+}\right),\left(8^{+}\right),\left(7^{-}\right)$and $5^{-}$two-proton-hole states, their excitation energies, and their dominant configurations.

As the two removed nucleons are assumed to be uncorrelated, and assuming that the valence proton structure in ${ }^{208} \mathrm{~Pb}$ is $[j]^{N}$, several results follow. The first is that the calculated (unit) cross sections for removal of a proton pair are as given in Table 1. This sets the scale for the anticipated cross sections. Based on an assumed $[j]^{N}$ configuration this predicts an inclusive cross section of $N(N-1) \sigma_{j j}(J) / 2$. However, it also follows that this cross section yield will be shared between different core final states with associated strengths $S\left(J^{\pi}\right)$. For removal of a pair from a $\left|[j]^{N}, 0^{+}\right\rangle$occupied sub-shell, $N$ even, the inclusive cross section will be distributed between final states $J^{\pi}$ according to the square of the coefficients of fractional parentage and, explicitly [15],

$$
S\left(J^{\pi} \neq 0^{+}\right)=\frac{N(N-1)}{2}\left[\frac{2(N-2)}{(N-1)} \frac{(2 J+1)}{(2 j-1)(2 j+1)}\right],
$$

where $\sum_{J} S\left(J^{\pi}\right)=N(N-1) / 2$. This yields $S\left(10^{+}\right)=21, S\left(8^{+}\right)=17$, being $S\left(J^{\pi}\right)=$

TABLE 1. Calculated two-proton removal cross sections from ${ }^{208} \mathrm{~Pb}$ at $1.0 \mathrm{GeV} /$ nucleon. The theoretical stripping cross sections, $\sigma_{j_{1} j_{2}}(J)$ [Eq. (2)] and the total diffractive contributions, $\tilde{\sigma}_{j_{1} j_{2}}(J)$, are shown. Also shown are the scaling factors $S\left(J^{\pi}\right)$ for each $J^{\pi}$ final state and the resulting theoretical partial cross sections $\sigma_{J}$.

\begin{tabular}{cccccc}
\hline$J^{\pi}$ & 2p configuration & $\sigma_{j_{1} j_{2}}(J)(\mathrm{mb})$ & $\tilde{\sigma}_{j_{1} j_{2}}(J)(\mathrm{mb})$ & $S\left(J^{\pi}\right)$ & $\sigma_{J}(\mathrm{mb})$ \\
\hline$\left(10^{+}\right)$ & {$\left[0 h_{11 / 2}\right]^{2}$} & 0.0082 & 0.0016 & 21 & 0.21 \\
$\left(8^{+}\right)$ & {$\left[0 h_{11 / 2}\right]^{2}$} & 0.0073 & 0.0015 & 17 & 0.15 \\
$\left(7^{-}\right)$ & {$\left[0 h_{11 / 2}, 1 d_{3 / 2}\right]$} & 0.0153 & 0.0038 & 15 & 0.29 \\
$5^{-}$ & {$\left[0 h_{11 / 2}, 2 s_{1 / 2}\right]$} & 0.0133 & 0.0034 & 11 & 0.18 \\
\hline
\end{tabular}


$2 J+1$, due to the filled $\left[0 h_{11 / 2}\right]^{12}$ sub-shell, and with $\sum_{J} S\left(J^{\pi}\right)=66$. Thus, in excess of half the inclusive cross section from proton pair removal from the $\left[0 h_{11 / 2}\right]^{12}$ sub-shell is expected to populate the (seniority-2) $\left(10^{+}\right)$and $\left(8^{+}\right)$final states.

The corresponding inclusive cross section for knockout of a pair from different subshells, e.g. a $\left[j_{1}\right]^{N_{1}}\left[j_{2}\right]^{N_{2}}$ configuration, is $N_{1} N_{2} \sigma_{j_{1} j_{2}}(J)$. The analogous distribution of this strength among $J^{\pi}$ final states is also discussed in [15]. For the case of protons removed from a pair of filled sub-shells, as arises for both the $\left(7^{-}\right)\left(\left[0 h_{11 / 2}\right]^{12}\left[1 d_{3 / 2}\right]^{4}\right)$ and $5^{-}\left(\left[0 h_{11 / 2}\right]^{12}\left[2 s_{1 / 2}\right]^{2}\right)$ states, these are simply $S\left(J^{\pi}\right)=(2 J+1)$. These weights were used to compute the $\left(10^{+}\right)(3.723 \mathrm{MeV}),\left(8^{+}\right)(3.623 \mathrm{MeV}),\left(7^{-}\right)(2.466 \mathrm{MeV})$ and $5^{-}(2.102 \mathrm{MeV})$ direct two-proton removal cross sections, shown in Table 1.

\section{SUMMARY AND CONCLUSIONS}

We have calculated two-proton removal cross sections, from ${ }^{208} \mathrm{~Pb}$ at $1.0 \mathrm{GeV} /$ nucleon on a ${ }^{9} \mathrm{Be}$ target, populating the assumed $\left(10^{+}\right)(3.723 \mathrm{MeV}),\left(8^{+}\right)(3.623 \mathrm{MeV}),\left(7^{-}\right)$ $(2.466 \mathrm{MeV})$ and $5^{-}(2.102 \mathrm{MeV})$ seniority-2, two-proton hole states in ${ }^{206} \mathrm{Hg}$. This important (test) case predicts significant cross sections from the direct two-proton knockout mechanism and a ratio of the isotopic yields for the $\left(10^{+}\right): 5^{-}$states of $0.21: 0.83$. This ratio is in line with very preliminary experimental indications [16]. Other systems of interest, and with final states expected to be populated cleanly via the direct two-proton knockout mechanism, include $\pi\left[0 g_{7 / 2}\right]^{2}$ and $\pi\left[0 h_{11 / 2}\right]^{2}$ proton-pair removal from ${ }^{136} \mathrm{Xe}$ and a ${ }^{206} \mathrm{Hg}$ secondary beam, respectively.

The financial support of the United Kingdom Engineering and Physical Sciences Research Council (EPSRC) through Grant No. EP/D003628, and extended discussions with Drs P.H. Regan, Zs. Podolyák, and P.D. Stevenson are gratefully acknowledged.

\section{REFERENCES}

1. P.G. Hansen and J.A. Tostevin, Annu. Rev. Nucl. Part. Sci. 53, 219 (2003).

2. D. Bazin et al., Phys. Rev. Lett. 91, 012501 (2003).

3. J.A. Tostevin, G. Podolyák, B.A. Brown, and P.G. Hansen, Phys. Rev. C 70, 064602 (2004).

4. J. Fridmann et al., Nature 435, 922 (2005).

5. A. Gade, R.V.F. Janssens et al., in preparation.

6. K. Yoneda et al., in preparation.

7. J.A. Tostevin, Diffractive contributions to direct two-nucleon knockout reactions, in preparation.

8. R.J. Glauber, in Lectures in Theoretical Physics, ed. by W.E. Brittin (Interscience, New York, 1959), Vol.1, p315.

9. J.A. Tostevin, Nucl. Phys. A 682, 320c (2001).

10. L. Ray, Phys. Rev. C 20, 1857 (1979).

11. P.D. Stevenson, private communication, 2005.

12. J. Dobaczewski, H. Flocard and J. Treiner, Nucl. Phys. A 422, 103 (1984).

13. A. Gade et al., Phys. Rev. Lett. 93, 042501 (2004).

14. B. Fornal et al., Phys. Rev, Lett. 87, 212501 (2001).

15. Norman K. Glendenning, Phys. Rev. 137, B102 (1965).

16. Zs. Podolyák et al., private communication, 2005. 